\title{
Synthesizing Global Exceptional Patterns in Different Data Sources
}

\author{
Animesh Adhikari
}

\begin{abstract}
Many large companies transact from multiple branches. It results in generating multiple databases, since local transactions are stored locally. The number of multi-branch companies as well as the number of branches of a multi-branch company is increasing over time. Thus, it is important to study data mining on multiple databases. Global exceptional patterns describe interesting individuality of few branches. Therefore, it is interesting to identify such patterns. In this paper, we propose type I and type II global exceptional frequent itemsets in multiple databases by extending the notion of global exceptional frequent itemset. Also, we propose the notion of exceptional sources for a type II global exceptional frequent itemset. We propose type I and type II global exceptional association rules in multiple databases by extending the notion of global exceptional association rule. We propose an algorithm for synthesizing type II global exceptional frequent itemsets. Experimental results are presented on both real and synthetic databases. We compare the proposed algorithm with the existing algorithm theoretically as well as experimentally. The experimental results show that the proposed algorithm is effective and promising.
\end{abstract}

Keywords. Global Exceptional Association Rule, Global Exceptional Pattern, Multi-Database Mining, Synthesis of Pattern.

2010 Mathematics Subject Classification. 68T10.

\section{Introduction}

Many multi-branch companies transact from different locations. They collect transactional data continuously through different branches. Due to a growthoriented and liberal economic policy adopted by many countries across the globe, the number of such companies as well as the number of branches of a company is increasing over time. Thus, there is an urgent need of data analyses on multiple databases. The first question comes to our mind whether a traditional data mining technique could deal with the multiple large databases. To apply a traditional data mining technique, we need to amass all the databases together. A single computer may take an unreasonable amount of time to process the entire database. Sometimes it might not be feasible to carry out a mining task. Another solution would be to employ parallel machines. It requires high investment on hardware 
and software. Moreover, it is difficult to identify local patterns when a traditional data mining technique is applied to the entire database. Thus, the traditional data mining techniques are not suitable in this situation. It seems mining multiple large databases is a different problem. Hence, it is required to be dealt with in a different way. One could employ the model of local pattern analysis [31] for mining multiple large databases. Under this model, each branch requires mining the local database using a traditional data mining technique. Afterwards, each branch forwards the pattern base to the central office. Then the central office processes the local pattern bases for synthesizing the global patterns, or making global decisions.

Based on the number of data sources, the patterns in multiple databases could be classified into the following categories: local patterns, global patterns and patterns that are neither local nor global. The third category of patterns is based on a few branch databases of a multi-branch company. For example, Asia-specific patterns could be based on the branch databases originated from Asia. A pattern based on a single database is called a local pattern. Local patterns are useful for local data analysis. But global patterns are based on all the databases under consideration. They are useful for global data analyses $[4,27]$ and global decision making problems $[2,26]$. In many data mining applications, we deal with various types of patterns such as frequent itemsets [8], positive associative rules [8] and conditional patterns [3]. There is no fixed set of attributes to describe different types of patterns. Each type of pattern could be described by a specific set of attributes. In general, it might be difficult to define a pattern in a database by using certain attributes.

Itemset patterns influence KDD research heavily in the following ways: Firstly, many interesting algorithms have been reported on mining itemset patterns in a database [10,17,21]. Secondly, an itemset could be considered as a basic type of pattern in a transactional database, since many patterns are derived from the itemset patterns. Some examples of derived patterns are negative association rule [25], high frequency association rule [27] and heavy association rule [4]. A considerable amount of work has been reported on mining/synthesizing such derived patterns in databases. Thirdly, solutions to many problems are based on the analysis of patterns in a database. Such applications process patterns in a database for the purpose of making some decisions $[2,22,26]$. Thus, mining and analysis of itemset patterns in a database is an interesting as well as important issue.

We discuss here a few concepts and notations that are used in this paper. The support [8] of an itemset $X$ in database $D$, denoted by $\operatorname{supp}(X, D)$, could be defined as the fraction of transactions in $D$ containing all the items of $X$. In most of the cases, the importance of an itemset is judged by its support. Itemset $X$ is frequent in $D$ if $\operatorname{supp}(X, D) \geq \alpha$, where $\alpha$ is user-defined level of minimum support. Let $\operatorname{SFIS}(D)$ be the set of frequent itemsets in database $D$. Frequent 
itemsets determine major characteristics of a database. Wu et al. [24] proposed a solution to inverse frequent itemset mining. They argued that one could efficiently generate a synthetic market basket dataset from the frequent itemsets and their supports. Let $X$ and $Y$ be two itemsets in $D$. The characteristics of $D$ are revealed more by the pair $(X, \operatorname{supp}(X, D))$ than that of $(Y, \operatorname{supp}(Y, D))$, if $\operatorname{supp}(X, D)>$ $\operatorname{supp}(Y, D)$. Thus, it is important to study frequent itemsets more than infrequent itemsets. In this paper, we propose two types of exceptional patterns in multiple databases, and an algorithm for synthesizing such patterns.

The rest of the paper is organized as follows. In Section 2, we propose different exceptional patterns in multiple databases. In this section, we also discuss other exceptional patterns that are reported in conjunction with multi-database mining. Section 3 presents the problem formally. We discuss related work in Section 4. We provide the justification of the proposed work in Section 5. In Section 6, we present a technique for synthesizing support of an itemset. Section 7 presents an algorithm for synthesizing type II global exceptional itemsets in multiple databases. We define two types of error in Section 8. Experimental results are presented in Section 9. Finally, we conclude the paper in Section 10.

\section{Exceptional Patterns}

In this section, we propose type I and type II global exceptional frequent itemsets as well as type I and type II global exceptional association rules in multiple databases. We also discuss other exceptional patterns that are reported in the literature. We propose different notions required before proposing the notion of an exceptional pattern. Thus, the definition of an exceptional pattern has been built incrementally. The difficulty of synthesizing a type II global exceptional frequency itemsets is that a frequent itemset in multiple databases may not get reported from every local database. Apart from synthesized support of an itemset, the number of extractions of the itemset is an important issue. An itemset may have high frequency or, low frequency, or neither high frequency nor low frequency. In the context of type II global exceptional patterns, we may need to consider only low frequency itemsets. One could arrive in a conclusion only if there is a predefined threshold of minimum number of extractions. Thus, a type II global exceptional frequency itemset in multiple databases could be judged against two thresholds, viz., high support and low extraction. Let $\gamma_{1}$ and $\gamma_{2}$ be the thresholds of low and high extractions of an itemset respectively, $0<\gamma_{1}<\gamma_{2} \leq 1$. Low and high frequency itemsets are defined as follows. 
Definition 1. Let $X$ be an itemset extracted from $k$ out of $n$ data sources.

(a) $X$ has low frequency, if $k<n \times \gamma_{1}$, where $\gamma_{1}$ is the user-defined threshold of low extraction.

(b) $X$ has high frequency, if $k \geq n \times \gamma_{2}$, where $\gamma_{2}$ is the user-defined threshold of high extraction.

Among low frequency itemsets, we shall search for type II global exceptional frequency itemsets. An itemset may not get extracted from all the databases. Sometimes we need to estimate the support of an itemset in a local database to synthesize its support in $D$. Let $\operatorname{supp}_{a}\left(X, D_{i}\right)$ be the actual support of an itemset $X$ in $D_{i}, i=1,2, \ldots, n$. Let $\mu_{1}$ and $\mu_{2}$ be the thresholds of low and high supports for an itemset in a database respectively, $0 \leq \mu_{1}<\alpha<\mu_{2} \leq 1$. For a single database, we define an itemset with high or low support as follows:

Definition 2. Let $X$ be an itemset in database $D_{i}, i=1,2, \ldots, n$.

(a) $X$ possesses high support in $D_{i}$ if $\operatorname{supp}_{a}\left(X, D_{i}\right) \geq \mu_{2}$, where $\mu_{2}(>\alpha)$ is the user-defined threshold of high support, $i=1,2, \ldots, n$.

(b) $X$ possesses low support in $D_{i}$ if $\operatorname{supp}_{a}\left(X, D_{i}\right)<\mu_{1}$, where $\mu_{1}(<\alpha)$ is the user-defined threshold of low support, $i=1,2, \ldots, n$.

The method of synthesizing support of an itemset is discussed in Section 7. Let $\operatorname{supp}_{s}(X, D)$ be the synthesized support of the itemset $X$ in $D$. In multi-database mining environment, we define an itemset with high/low support as follows:

Definition 3. Let $D$ be the union of all branch databases.

(a) An itemset $X$ in $D$ possesses high support if $\operatorname{supp}_{s}(X, D) \geq \mu_{2}$, where $\mu_{2}$ is the user-defined threshold of high support.

(b) An itemset $X$ in $D$ possesses low support if $\operatorname{supp}_{s}(X, D)<\mu_{1}$, where $\mu_{1}$ is the user-defined threshold of low support.

Based on the concepts stated above, we propose type II global exceptional frequent itemset in $D$ as follows:

Definition 4. Let $D$ be the union of all branch databases. Let $X$ be a frequent itemset in some branch databases. Then $X$ is a type II global exceptional itemset in $D$ if it has low frequency and high support in $D$.

In a similar manner, we propose type I global exceptional frequent itemset in $D$ as follows: 
Definition 5. Let $D$ be the union of all branch databases. Let $X$ be a frequent itemset in some branch databases. Then $X$ is a type I global exceptional itemset in $D$ if it has high frequency and low support in $D$.

Association rule mining has received a lot of attention in the KDD community $[7,25,29,30]$. It is based on the support-confidence framework established by Agrawal et al. [8]. Let $I$ be set of items in database $D_{1}$. An association rule $r$ has been expressed symbolically as $X \rightarrow Y$, where $X=\left\{x_{1}, x_{2}, \ldots, x_{p}\right\}$, and $Y=\left\{y_{1}, y_{2}, \ldots, y_{q}\right\} ; x_{i}, y_{j} \in I, i=1,2, \ldots, p, j=1,2, \ldots, q$. It expresses association between the itemsets $X$ and $Y$, called the antecedent and consequent, of $r$ respectively. The meaning attached to this implication could be expressed as follows. If the items in $X$ are purchased by a customer then the items in $Y$ are likely to be purchased by the same customer at the same time. The interestingness of an association rule could be expressed by its support and confidence. The support and confidence of association rule $r$ in database $D_{1}$ could be expressed as follows: $\operatorname{supp}_{a}\left(r, D_{1}\right)=\operatorname{supp}_{a}\left(X Y, D_{1}\right)$, and $\operatorname{conf}_{a}\left(r, D_{1}\right)=$ $\operatorname{supp}_{a}\left(X Y, D_{1}\right) / \operatorname{supp}_{a}\left(X, D_{1}\right)$. One needs to deal with synthesized support $\left(\operatorname{supp}_{s}\right)$ and synthesized confidence $\left(\operatorname{conf}_{s}\right)$ of an association rule, since actual support and actual confidence of an association rule in multiple databases might not be available. There exist many techniques by which one could synthesize support of an itemset. Using pipelined feedback technique [6] one could obtain a good estimate of support of an itemset in multiple databases. An association rule $r$ in $D_{1}$ is interesting if $\operatorname{supp}_{a}\left(r, D_{1}\right) \geq \operatorname{minimum} \operatorname{support}(\alpha)$, and $\operatorname{conf}_{a}\left(r, D_{1}\right) \geq$ minimum confidence $(\beta)$. Here the parameters $\alpha$ and $\beta$ are userdefined. In the following we present the concept of heavy association rule in multiple databases. First, we present the concept of heavy association rule in a single database. Afterwards, we shall present the concept of heavy association rule in multiple databases.

Definition 6. An association rule $r$ in database $D_{1}$ is heavy if $\operatorname{supp}_{a}\left(r, D_{1}\right) \geq \mu$, and $\operatorname{conf}_{a}\left(r, D_{1}\right) \geq v$, where $\mu(>\alpha)$ and $v(>\beta)$ are the user-defined thresholds of high support and high confidence for identifying heavy association rules in $D_{1}$ respectively.

If an association rule is heavy in a local database then it might not be heavy in the union of all databases $D$. An association rule in $D$ might have different statuses in different local databases. For example, it might be a heavy association rule, or an association rule, or absent in a local database. Thus, one needs to synthesize an association rule for determining its overall status in $D$. A method of synthesizing an association rule is discussed in [4]. After synthesizing an association rule, we 
get synthesized support and synthesized confidence of the association rule in $D$. Now, we present the concept of heavy association rule in $D$ as follows.

Definition 7. Let $D$ be the union of local databases. An association rule $r$ in $D$ is heavy if $\operatorname{supp}_{s}(r, D) \geq \mu$, and $\operatorname{conf}_{s}(r, D) \geq v$, where $\mu$ and $v$ are the user-defined thresholds of high support and high confidence for identifying heavy association rules in $D$ respectively.

Apart from synthesized support and synthesized confidence of an association rule, the frequency of an association rule is an important issue in multi-database mining. We define the frequency of an association rule as the number of extractions of the association rule from different data sources. If an association rule is extracted from $k$ out of $n$ databases then the frequency of the association rule is $k, 0 \leq k \leq n$. An association rule may have low frequency or, high frequency or, neither high frequency nor low frequency in multiple data sources. One could arrive in such a conclusion only if we have user-defined thresholds of low frequency $\left(\gamma_{1}\right)$, and high frequency $\left(\gamma_{2}\right)$ of an association rule, $0<\gamma_{1} \leq \gamma_{2} \leq 1$. Earlier we have discussed user-defined parameters $\gamma_{1}$ and $\gamma_{2}$ while defining type I and type II global exceptional frequent itemsets. In multi-database mining using local pattern analysis, the concepts of high frequency association rule and low frequency association rule are presented as follows [4].

Definition 8. Let an association rule be extracted from $k$ out of $n$ data sources.

(a) The association rule has low frequency if $k<n \times \gamma_{1}$, where $\gamma_{1}$ is the userdefined threshold of low frequency.

(b) The association rule has high frequency if $k \geq n \times \gamma_{2}$, where $\gamma_{2}$ is the userdefined threshold of high frequency.

While synthesizing heavy association rules in multiple databases, it may be worth noting the other attributes of a synthesized association rule. For example, high frequency, low frequency, and exceptionality are interesting as well as important attributes of a synthesized association rule. Similar to Definitions 4 and 5, we propose type I and type II global exceptional association rules in multiple data sources as follows.

Definition 9. A high frequency association rule in multiple databases is type I global exceptional if it has low support.

Definition 10. A heavy association rule in multiple databases is type II global exceptional if it has low frequency. 
Adhikari et al. [5] proposed an iceberg pattern in multiple time-stamped databases as a generalized notch that satisfies the following conditions: (i) The height of the generalized notch is greater than or equal to $\alpha$, and (ii) the width of the generalized notch is greater than or equal to $\beta$. Both $\alpha$ and $\beta$ are user-defined thresholds. Adhikari et al. proposed an algorithm for mining icebergs in multiple time-stamped databases.

\section{Problem Statement}

Let $X$ be a type II global exceptional frequent itemset in $D$. Without any loss of generality, let $X$ be extracted from $D_{1}, D_{2}, \ldots, D_{k}, 1 \leq k \leq n$. Let the actual support of $X$ in $D_{i}$ be $\operatorname{supp}_{a}\left(X, D_{i}\right), i=1,2, \ldots, k$. Then the average of these supports is obtained by the following formula:

$$
\operatorname{avg}\left(\operatorname{supp}(X), D_{1}, D_{2}, \ldots, D_{k}\right)=\left(\sum_{i=1}^{k} \operatorname{supp}_{a}\left(X, D_{i}\right)\right) / k .
$$

Database $D_{i}$ is called an exceptional source with respect to the type II global exceptional frequent itemset $X$, if $\operatorname{supp}_{a}\left(X, D_{i}\right) \geq \operatorname{avg}\left(\operatorname{supp}(X), D_{1}, D_{2}, \ldots, D_{k}\right)$, $i=1,2, \ldots, k$. Let us take an example to explain this issue. Let $X$ be a global exceptional frequent itemset in $D$, and it has been extracted from $D_{1}, D_{4}$, and $D_{7}$ out of ten data sources $D_{1}, D_{2}, \ldots, D_{10}$. Let $\operatorname{supp}_{a}\left(X, D_{1}\right)=0.09$, $\operatorname{supp}_{a}\left(X, D_{4}\right)=0.17$, and $\operatorname{supp}_{a}\left(X, D_{7}\right)=0.21$. Then,

$$
\operatorname{avg}\left(\operatorname{supp}(X), D_{1}, D_{4}, D_{7}\right)=(0.09+0.17+0.21) / 3=0.157 .
$$

The databases $D_{4}$ and $D_{7}$ are exceptional sources for type II global exceptional frequent itemset $X$, since $0.17>0.157$, and $0.21>0.157$. Now we state the problem as follows:

Let $D_{1}, D_{2}, \ldots, D_{n}$ be $n$ data sources, and $D$ the union of these data sources. Let $\operatorname{SFIS}\left(D_{i}\right)$ be the set of frequent itemsets in $D_{i}, i=1,2, \ldots, n$. Find the type II global exceptional frequent itemsets in $D$ using $\operatorname{SFIS}\left(D_{i}\right), i=1,2, \ldots, n$. Also, report the exceptional sources for each type II global exceptional frequent itemset in $D$.

\section{Related Work}

Multi-database mining has been recently recognized as an important research topic in data mining [1,32]. Zhang et al. [31] proposed local pattern analysis for mining multiple large databases. Afterwards, we proposed an extended model of local 
pattern analysis [4]. The extended model provides a way to mine and analyze multiple large databases approximately. Later, we proposed the pipelined feedback technique [6] for improving the accuracy of multi-database mining.

Distributed data mining (DDM) algorithms deal with mining multiple databases distributed over different geographical regions. In the last few years, researchers have started addressing problems where the databases stored at different places cannot be moved to a central storage area for a variety of reasons. A distributed data mining environment often comes with different distributed sources of computation. The advent of ubiquitous computing [16], sensor networks [34], grid computing [23], and privacy-sensitive multiparty data [19] present examples where centralization of data is either not possible, or at least not always desirable.

In the context of support estimation of frequent itemsets, Jaroszewicz and Simovici [18] proposed a method for estimating supports of frequent itemsets using Bonferroni-type inequalities [15]. Also, the maximum-entropy approach to support estimation of a general Boolean expression is proposed by Pavlov et al. [20]. But, these support estimation techniques might be suitable for problems that deal with single database.

Existing parallel mining techniques $[9,11,12]$ could also be used to deal with multiple databases. Parallel algorithms are also designed with a different objective. They provide a costly solution to multi-databases mining. It might require high investment on hardware and software. One should make a cost-benefit analysis before implementing such a decision. In many situations, it might not be an acceptable solution to the management of the company. Moreover, it might be difficult to find regional patterns when a traditional data mining technique is applied to the entire dataset.

\section{Justification of the Proposed Work}

Consider a multi-branch company with $n$ branches. Let $D_{i}$ be the database corresponding to the $i$-th branch, $i=1,2, \ldots, n$. Also, let $D$ be the union of the branch databases. In the context of multiple databases, one could think of global exceptional patterns in two ways: (i) A pattern that has been extracted from the most of the databases, but has a low support in $D$ (type I exceptional pattern). (ii) A pattern that has been extracted from a few databases, but has a high support in $D$ (type II exceptional pattern). Both types of exceptional patterns are global in nature, since all the branch databases are considered. In this paper, we are interested in mining type II exceptional patterns. Zhang et al. [33] proposed a strategy for mining local exceptions in multiple databases. They defined an exceptional pattern as follows: 
A pattern $p$ in local instances is an exceptional pattern if $\operatorname{EPI}(p) \geq \operatorname{minEP}$, where $\operatorname{EPI}(p)$ is an interestingness measure of $p$ and has been defined as follows:

$$
\operatorname{EPI}(p)=\frac{\operatorname{nExtrn}(p)-\operatorname{avgNoExtrn}}{-\operatorname{avgNoExtrn}},
$$

where $n \operatorname{Extrn}(p)$ and avgNoExtrn are the number of times $p$ gets extracted and the average number of times a pattern gets extracted from different data sources, respectively. minEP is the user-defined threshold of minimum interest degree. Also, Zhang et al. defined interestingness of a pattern in a branch as follows:

A pattern $p$ in $i$-th branch is of interest if $\mathrm{RI}_{i}(p) \geq \operatorname{minEPsup}$, where $\mathrm{RI}_{i}(p)$ is the interestingness degree of $p$ in the $i$-th branch defined as

$$
\mathrm{RI}_{i}(p)=\left(\operatorname{supp}\left(p, D_{i}\right)-\alpha_{i}\right) / \alpha_{i},
$$

where $\alpha_{i}$ is the minimum support given for mining $D_{i}, i=1,2, \ldots, n$; minEPsup is the user-defined threshold for minimum interest degree.

From the above two definitions, we observe the following points:

(i) The definition of exceptional pattern is considered with respect to all the databases. The definition of interestingness of a pattern is considered with respect to a local database. Thus, an exceptional pattern in multiple databases and interestingness of the pattern in a local branch are of two different issues.

(ii) For a pattern $p$ in local instances, Zhang et al. showed that $0<\operatorname{EPI}(p) \leq 1$. We take the following example to show that the above property does not hold always. Let there be only four patterns $p_{1}, p_{2}, p_{3}$, and $p_{4}$ in 15 databases. The number of extractions of these patterns are given as follows: $\operatorname{nExtrn}\left(p_{1}\right)=2$, $\operatorname{nExtrn}\left(p_{2}\right)=15, \mathrm{nExtrn}\left(p_{3}\right)=4, n \operatorname{Extrn}\left(p_{4}\right)=5$. Thus,

$$
\begin{aligned}
\operatorname{avgNoExtrn} & =26 / 4=6.5, \\
\operatorname{EPI}\left(p_{1}\right) & =(2-6.5) /(-6.5)=0.69, \\
\operatorname{EPI}\left(p_{2}\right) & =(15-6.5) /(-6.5)=-1.31, \\
\operatorname{EPI}\left(p_{3}\right) & =(4-6.5) /(-6.5)=0.38, \\
\operatorname{EPI}\left(p_{4}\right) & =(5-6.5) /(-6.5)=0.23 .
\end{aligned}
$$

Thus, $\operatorname{EPI}\left(p_{2}\right) \notin(0,1]$.

(iii) An interesting exceptional pattern might not emerge as a global exceptional pattern, since the support of the pattern is not considered in the union of all databases. It is reasonable that an exceptional frequency itemset should be constrained on the number of times it gets extracted, and its support in the union of all 
databases. Thus, none of the above two definitions, nor both definitions together serve as a definition of exceptional frequency itemset in multiple databases.

Zhang et al. [28] proposed a technique for identifying global exceptional patterns in multiple databases. They described global exceptional pattern as follows:

Global exceptional patterns are highly supported by only a few branches, that is to say, these patterns have very high support in these branches and zero support in other branches.

From the above descriptions, we observe the following:

Let there be ten branches of a multi-branch company. A pattern $p$ has very high support in the first two databases that have small sizes. Also, $p$ does not get extracted from the remaining databases. According to the above description, $p$ is a global exceptional pattern. We observe that pattern $p$ might not have high support in the union of all databases. Thus, such description does not serve the purpose. Also, it is not necessarily true that a type II exceptional pattern will have zero support in the remaining databases. Thus, the above description does not describe type II global exceptional patterns in true sense. Also, we observe the following points in the IdentifyExPattern algorithm [28] for identifying exceptional patterns in multiple databases:

(i) We believe that the size (i.e., the number of transactions) of a database and support of an itemset in the database are two important parameters for determining the presence of an itemset in a database, since the number of transactions containing the itemset $X$ in a database $D_{1}$ is equal to $\operatorname{supp}\left(X, D_{1}\right) \times \operatorname{size}\left(D_{1}\right)$. The algorithm does not consider the size of a database to synthesize the global support of a pattern. Global support of a pattern has been synthesized using only supports of the pattern in the individual databases. We take the following example to illustrate this issue. Let there be two databases $D_{1}$ and $D_{2}$, where $\operatorname{size}\left(D_{1}\right)$ is significantly greater than size $\left(D_{2}\right)$. At a given $\alpha$, we assume that pattern $p$ does not get extracted from $D_{2}$, and pattern $q$ does not get extracted from $D_{1}$. Thus, $\operatorname{supp}\left(p, D_{2}\right)$ and $\operatorname{supp}\left(q, D_{1}\right)$ both are assumed as 0. Then, $\operatorname{supp}\left(p, D_{1} \cup D_{2}\right)$ could be synthesized by

$$
\left[\operatorname{supp}\left(p, D_{1}\right) \times \operatorname{size}\left(D_{1}\right)+0 \times \operatorname{size}\left(D_{2}\right)\right] / \operatorname{size}\left(D_{1} \cup D_{2}\right) .
$$

If $\operatorname{supp}\left(p, D_{1}\right)<\operatorname{supp}\left(q, D_{2}\right)$, then it might happen that $\operatorname{supp}\left(p, D_{1} \cup D_{2}\right)>$ $\operatorname{supp}\left(q, D_{1} \cup D_{2}\right)$. In particular, let size $\left(D_{1}\right)=10,000$, $\operatorname{size}\left(D_{2}\right)=100$. At $\alpha=0.05$, let $\operatorname{supp}\left(p, D_{1}\right)=0.1, \operatorname{supp}\left(q, D_{1}\right)=0, \operatorname{supp}\left(p, D_{2}\right)=0$, and $\operatorname{supp}\left(q, D_{2}\right)=0.2$. We note that $\operatorname{supp}\left(p, D_{1}\right)<\operatorname{supp}\left(q, D_{2}\right) . \operatorname{But}, \operatorname{supp}(p$, $\left.D_{1} \cup D_{2}\right)=0.099$, and $\operatorname{supp}\left(q, D_{1} \cup D_{2}\right)=0.002$. So, $\operatorname{supp}\left(p, D_{1} \cup D_{2}\right)>$ $\operatorname{supp}\left(q, D_{1} \cup D_{2}\right)$. Thus, the size of a database is an important parameter to synthesize the support of a pattern in the union of all databases. 
(ii) The algorithm does not identify type II global exceptional patterns correctly in all the situations. For example, let there be 10 similar databases. Assume that the number of times each pattern gets extracted is either 8 , or 9 , or 10 . Thus, these patterns are supported by most of the databases. According to the nature of type II global exceptional patterns, a high voted pattern is not a type II global exceptional pattern. But, the algorithm would report some of them as type II global exceptional patterns.

(iii) The algorithm returns patterns that have high supports among the patterns that are extracted less than the average number of times. It is reasonable that a type II global exceptional pattern should have the following properties: (a) the support of a type II global exceptional pattern in the union of all databases is greater than or equal to a user-defined threshold, and (b) the number of extractions of a type II global exceptional pattern is less than a user-defined threshold. Thus, the motivations of this paper are to propose the definition of different global exceptional patterns and design an algorithm to mine them. Secondly, there are useful applications of exceptional frequency itemsets. For example, a company might plan to collect the feedback of customers for the exceptional products and implement strategies to increase their sales. Also, the company could identify the branches having high sales of the exceptional items. It might plan to manufacture and/or procure such items locally to reduce transportation cost. Thus, exceptional frequency items might affect many decisions of a multi-branch company.

\section{Synthesizing Support of an Itemset}

Synthesizing support of an itemset in multiple databases is an important issue. First we present here the support estimation technique proposed in [4]. In real databases, the trend of the customers' behavior exhibited in one database is usually present in other databases. In particular, a frequent itemset in a database is usually present in some transactions in other databases even if it does not get reported. The estimation procedure captures such trend and estimates the support of an itemset that fails to get reported in a database. The estimated support of a missing itemset usually reduces the error of synthesizing a frequent itemset in multiple databases. If an itemset $X$ fails to get reported from database $D_{1}$, then we assume that $D_{1}$ contributes some amount of support to $X$. The support of $X$ in $D_{1}$ satisfies the following inequality: $0 \leq \operatorname{supp}_{a}\left(X, D_{1}\right)<\alpha$. The procedure of finding an estimated support, called average low-support (als), of an itemset is discussed below.

Let the itemset $X$ be reported from $m$ databases, $1 \leq m<n$. Without any loss of generality, we assume that $X$ has been extracted from the first $m$ databases. 
We shall use the average behavior of the customers of the first $m$ branches to estimate the average behavior of the customers in remaining branches. Let $D_{1, m}$ be the union of $D_{1}, D_{2}, \ldots, D_{m}$. Then $\operatorname{supp}_{a}\left(X, D_{1, m}\right)$ could be viewed as the average behavior of the customers of the first $m$ branches with respect to $X$. Thus, $\operatorname{supp}_{a}\left(X, D_{1, m}\right)$ could be obtained by the following formula.

$$
\operatorname{supp}_{a}\left(X, D_{1, m}\right)=\left(\sum_{i=1}^{m} \operatorname{supp}_{a}\left(X, D_{i}\right) \times \operatorname{size}\left(D_{i}\right)\right) / \sum_{i=1}^{m} \operatorname{size}\left(D_{i}\right) .
$$

One could estimate the support of $X$ for each of the remaining databases as

$$
\operatorname{als}\left(X, D_{i}\right)=\alpha \times \operatorname{supp}_{a}\left(X, D_{1, m}\right), \quad i=m+1, m+2, \ldots, n .
$$

The technique discussed above might not be suitable for synthesizing type II global exceptional frequent itemsets. The reason is given as follows. A type II global exceptional frequent itemset $X$ gets extracted from a few databases. During the process of synthesis, we need to estimate the supports of $X$ for the remaining databases. So, the number of actual supports of $X$ is less than the number of estimated supports of $X$. Thus, the error of synthesizing the support of $X$ in $D$ might be high. Thus, we shall follow a different strategy for synthesizing support of an itemset in $D$. The strategy is explained as follows. We shall mine databases at a reasonably low value of $\alpha$. If the itemset $X$ fails to get reported from $D_{i}$ then we assume that $\operatorname{supp}_{a}\left(X, D_{i}\right)=0$, for some $i$. In this case, we assume that the itemset $X$ is present in $D_{i}, i=1,2, \ldots, m$. Then the number of the transactions containing $X$ in $D_{i}$ is $\operatorname{supp}_{a}\left(X, D_{i}\right) \times \operatorname{size}\left(D_{i}\right), i=1,2, \ldots, m$. We assume that the estimated number of the transactions containing $X$ in $D_{i}$ is 0 , for $i=m+1, m+2, \ldots, n$. Thus, the estimated support of $X$ in a database is given as follows:

$$
\operatorname{supp}_{e}\left(X, D_{i}\right)= \begin{cases}\operatorname{supp}_{a}\left(X, D_{i}\right), & \text { for } i=1,2, \ldots, m, \\ 0, & \text { for } i=m+1, m+2, \ldots, n .\end{cases}
$$

The synthesized support of $X$ in $D$ can be obtained by the following formula:

$$
\operatorname{supp}_{s}(X, D)=\left(\sum_{i=1}^{n} \operatorname{supp}_{e}\left(X, D_{i}\right) \times \operatorname{size}\left(D_{i}\right)\right) / \sum_{i=1}^{n} \operatorname{size}\left(D_{i}\right) .
$$

\section{Synthesizing Type II Global Exceptional Itemsets}

In this section, we present an algorithm for synthesizing type II global exceptional frequent itemsets in $D$. We discuss here various data structures required to implement the proposed algorithm. Let $N$ be the number of frequent itemsets in 
$D_{1}, D_{2}, \ldots, D_{n}$. The frequent itemsets are kept in a two-dimensional array SFIS. The $(i, j)$-th element of SFIS stores the $j$-th frequent itemset extracted from $D_{i}$, $j=1,2, \ldots,|\operatorname{SFIS}(i)|, i=1,2, \ldots, n$. An itemset could be described by the following attributes: itemset, supp and did. Here the attributes itemset, supp and did represent itemset, support and database identification of the frequent itemset, respectively. Synthesized type II global exceptional frequent itemsets are kept in the array synFIS. Each type II global exceptional itemset has been described by the following attributes: itemset, ssupp, nSources, databases, nExSources, and exDbases. The attributes itemset and ssupp represent the itemset and synthesized support of the type II global exceptional frequent itemset in $D$, respectively. The attributes nSources and databases store the number of sources of exceptional frequent itemsets and the list of identifications of source databases for a type II global exceptional frequent itemset, respectively. The attributes nExSources and exDbases store the number of exceptional sources and the list of identifications of exceptional sources for a type II global exceptional frequent itemset, respectively.

Let us explain the algorithm presented in Algorithm 1. The frequent itemsets with the same itemset attribute are kept consecutive in array FIS. It helps processing one itemset at a time. A type II global exceptional frequent itemset is synthesized using the lines 6-31. The array sources is used to store the database identifications of all the databases that report the current frequent itemset. Also, the array supports is used to store the supports of the current frequent itemset in different databases. The information about the current itemset is obtained by the while-loop in lines 9-16. The information includes the number of extractions, database identifications of the source databases, supports in different databases, total supports, number of transactions containing current frequent itemset in different databases. We explain the update statement at line 11 as follows. The number of extraction of current frequent itemset, count, is increased by 1 . The database identification, did, of the current database is copied into cell sources(count). Variable nCurFIS is added by expression FIS $(i)$.supp $\times \operatorname{size}(\mathrm{FIS}(i)$.did $)$. Variable totalSupp is also added by expression FIS $(i)$.supp. The support of frequent itemset in the current database is copied into supports(count). We also explain the updatestatement at line 19 as follows. The synthesized support of current type II global exceptional frequent itemset is obtained by the expression nCurFIS/totTrans. The itemset attribute of current type II global exceptional frequent itemset is the same as the itemset attribute of current frequent itemset. The variable count is copied into synFIS(nSynFIS).nSources. The if-statement in lines 17-29 checks whether the current itemsets is a type II global exceptional frequent itemset in multiple databases, and it synthesizes each type II global exceptional frequent itemset. All the frequent itemsets are processed using the lines 5-31. We sort global exceptional itemsets for better presentation at line 32. All the type II global exceptional 


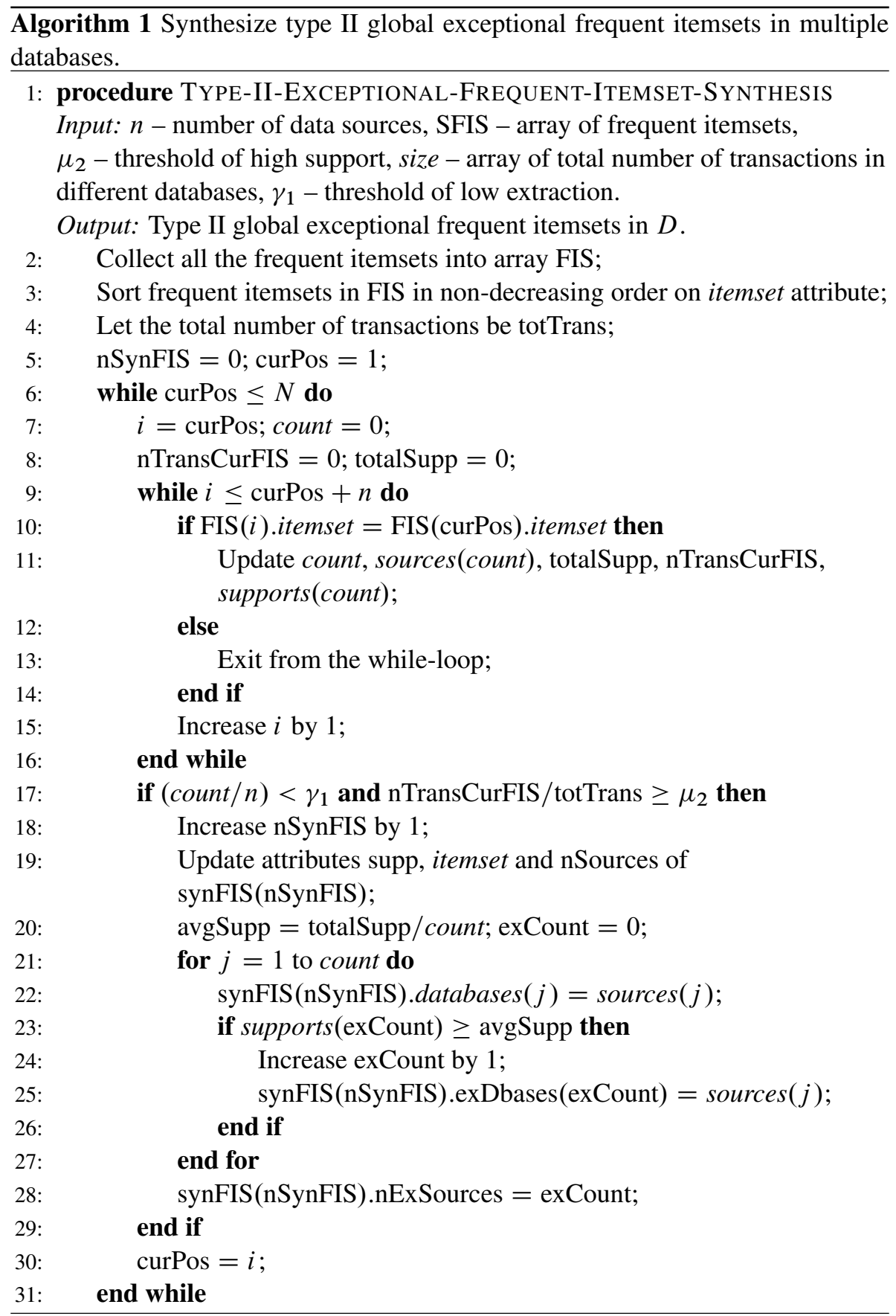




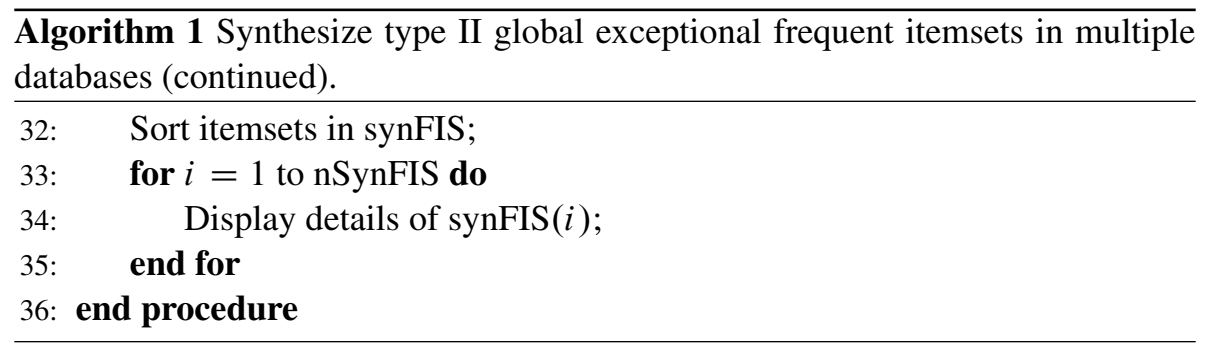

itemsets are kept in non-decreasing order on the length of the itemset. Again, the itemsets of the same length are sorted in non-increasing order on support of the itemset. Finally, type II global exceptional itemsets and supports are displayed using lines 33-35. For every type II global exceptional frequent itemset, we display the data sources from which it has been extracted. Also, for every type II global exceptional frequent itemset, we also display the exceptional data sources from which it has been highly supported.

Theorem 1. The time complexity of the procedure Type-II-Exceptional-FrequentItemset-Synthesis is $\max \{O(N \times \log (N)), O(n \times N)\}$, where $N$ is the number of frequent itemsets extracted from $n$ databases.

Proof. The time complexity of line 2 is $O(N)$, since there are $N$ frequent itemsets in $n$ local databases. Line 3 sorts $N$ frequent itemsets in $O(N \times \log (N))$ time. The time complexity of line 4 is $O(n)$, since there are $n$ databases. The program segment lines 6-31 repeats at most $N$ times. Within this program segment, there is a while-loop and a for-loop. The while-loop in lines 9-16 takes $O(n)$ time. Also, the for-loop in lines 21-27 takes $O(n)$ time. Thus, the time complexity of the program segment lines 6-31 is $O(n \times N)$. Line 32 takes $O(N \times \log (N))$ time for sorting at most $N$ synthesized type II global exceptional itemsets. To display the details of a type II global exceptional itemset it takes $O(n)$ time, since there are at most $n$ sources of the itemset. Thus, the program segment in lines 33-35 takes $O(n \times N)$ time. Therefore, the time complexity of the procedure Type-IIExceptional-Frequent-Itemset-Synthesis is $\max \{O(N \times \log (N)), O(n \times N)\}$.

We manually execute the above algorithm and verify that it works correctly with the help of Example 1. It is not a real one.

Example 1. Let $D_{1}, D_{2}$ and $D_{3}$ be three databases of 4,000, 3,290, and 10,200 transactions respectively. Let $D U$ be the union of $D_{1}, D_{2}$, and $D_{3}$. Assume that $\alpha=0.1, \gamma_{1}=0.4, \mu_{2}=0.25$. The sets of frequent itemsets extracted from these 
databases are given as follows:

$$
\begin{aligned}
& \operatorname{SFIS}\left(D_{1}\right)=\{A(0.12), B(0.14), A B(0.11), C(0.20)\}, \\
& \operatorname{SFIS}\left(D_{2}\right)=\{A(0.10), B(0.20), C(0.25), D(0.16), C D(0.12), E(0.16)\}, \\
& \operatorname{SFIS}\left(D_{3}\right)=\{A(0.11), C(0.60), F(0.77)\} .
\end{aligned}
$$

The symbol $X(\eta)$ is used to indicate that $X$ is a frequent itemset in the local database with support $\eta$. All the extracted itemsets have minimum support greater than or equal to 0.1 . Thus, infrequent local itemsets are not considered. $\gamma_{1}$ is the user-defined threshold of low extraction. The number of local databases is 3 . Here, $1 / 3=0.33$ and $2 / 3=0.67$. We assume that if an itemset gets extracted from less than two data sources, it becomes low voted. Otherwise, it becomes a high voted pattern. Therefore, $\gamma_{1}$ is assumed as a value in between $1 / 3$ and 2/3. $\mu_{2}$ is the threshold of high support. The value of high support is dependent on the context. Based on the assumed values of supports of different itemsets in Example 1, 0.25 is considered as a high support. So, we have taken $\mu_{2}$ equal to 0.25 . We keep frequent itemsets in the array FIS and sort them on itemset attribute. The sorted frequent itemsets are given in Table 1.

Here, totTrans is 17,490 . We synthesize the frequent itemsets in FIS. Synthesized frequent itemsets are given in Table 2.

In Algorithm 1, we maintain synthesized global exceptional frequent itemset in the array synFIS. For the purpose of explanation, Table 2 has been introduced here. From Table 2, we find that the synthesized supports of itemsets $C$ and $F$ are high, since $\operatorname{supp}_{s}(C, D U) \geq \mu_{2}$ and $\operatorname{supp}_{s}(F, D U) \geq \mu_{2}$. Itemset $F$ has been extracted from one out of three databases. Thus, $F$ is low-voted, since $1 / 3<\gamma_{1}$. Thus, the itemset $F$ is a type II global exceptional frequent itemset in $D U$.

\begin{tabular}{lccccccccccccc}
\hline itemset & $A$ & $A$ & $A$ & $B$ & $B$ & $C$ & $C$ & $C$ & $D$ & $E$ & $F$ & $A B$ & $C D$ \\
\hline supp & 0.12 & 0.10 & 0.11 & 0.14 & 0.20 & 0.20 & 0.25 & 0.60 & 0.16 & 0.16 & 0.77 & 0.11 & 0.12 \\
did & 1 & 2 & 3 & 1 & 2 & 1 & 2 & 3 & 2 & 2 & 3 & 1 & 2 \\
\hline
\end{tabular}

Table 1. Sorted frequent itemsets in different databases.

\begin{tabular}{lcccccccc}
\hline itemset & $A$ & $B$ & $C$ & $D$ & $E$ & $F$ & $A B$ & $C D$ \\
\hline synthesized support & 0.11 & 0.07 & 0.44 & 0.03 & 0.03 & 0.45 & 0.03 & 0.02 \\
\hline
\end{tabular}

Table 2. Synthesized frequent itemsets in multi-databases. 
In the following theorem, we determine the time complexity of the algorithm IdentifyExPattern [28] for comparing it with the proposed algorithm theoretically.

Theorem 2. The algorithm IdentifyExPattern takes $O\left(n^{2} \times N \times \log (N)\right)$ time, where $N$ is the number of frequent itemsets extracted from $n$ databases.

Proof. We refer to algorithm IdentifyExPattern [28]. Step 5 of the algorithm ranks candidate exceptional patterns based on their global supports. Step 4 calculates global support of a candidate exceptional pattern based on the number of databases that support the pattern. Step 1 counts the number of databases that support a specific pattern. Thus, Step 5 is based on Step 4, and Step 4 is based on Step 1. Step 1 takes $O(n)$ time for a specific pattern. This implies that Step 4 takes $O(n \times n)$ time for each candidate exceptional pattern. Thus, Step 5 takes $O\left(n^{2} \times N \times \log (N)\right)$ time, and hence the theorem follows.

From Theorems 1 and 2, we conclude that the proposed algorithm executes faster than algorithm IdentifyExPattern. We also compare these two algorithms experimentally in Section 9.

\section{Error of an Experiment}

To evaluate the proposed technique of synthesizing type II global exceptional frequent itemsets, we have measured the amount of error occurred in an experiment. Error of the experiment is relative to the number of transactions (i.e., the size of the database), number of items, and length of a transaction in a database. Thus, the error of the experiment needs to be expressed along with the ANT, ALT, and ANI in a database, where ANT, ALT, and ANI denote the average number of transactions, average length of a transaction, and the average number of items in a database, respectively. The error of the experiment is based on the global exceptional frequent itemsets in $D$. Let $\left\{X_{1}, X_{2}, \ldots, X_{m}\right\}$ be the set of type II global exceptional frequent itemsets in $D$. There are several ways one could define the error of an experiment. We have defined the following two types of error of an experiment.

1. Average error $(\mathrm{AE})$

$$
\operatorname{AE}(D, \alpha, \mu, \gamma)=\frac{1}{m} \sum_{i=1}^{m}\left|\operatorname{supp}_{a}\left(X_{i}, D\right)-\operatorname{supp}_{s}\left(X_{i}, D\right)\right| .
$$

2. Maximum error (ME)

$$
\operatorname{AE}(D, \alpha, \mu, \gamma)=\max \left\{\left|\operatorname{supp}_{a}\left(X_{i}, D\right)-\operatorname{supp}_{s}\left(X_{i}, D\right)\right|, i=1,2, \ldots, m\right\} .
$$


Actual support of $X_{i}$ in $D, \operatorname{supp}_{a}\left(X_{i}, D\right)$, is obtained by mining $D$ using a traditional data mining technique, $i=1,2, \ldots, m$. Synthesized support of $X_{i}$ in $D, \operatorname{supp}_{s}\left(X_{i}, D\right)$ is obtained by the technique presented in Section $6, i=$ $1,2, \ldots, m$. Then we compute the error of synthesizing support of $X_{i}$ in $D$ as $\left|\operatorname{supp}_{a}\left(X_{i}, D\right)-\operatorname{supp}_{s}\left(X_{i}, D\right)\right|, i=1,2, \ldots, m$.

Example 2. With reference to Example 1, the itemset $F$ is the only type II global exceptional frequent itemset present in $D$. Thus,

$$
\begin{aligned}
\operatorname{AE}(D, 0.1,0.25,0.4) & =\operatorname{ME}(D, 0.1,0.25,0.4) \\
& =\left|\operatorname{supp}_{a}(F, D)-\operatorname{supp}_{s}(F, D)\right| .
\end{aligned}
$$

\section{Experiments}

We have carried out several experiments to study the effectiveness of our approach. All the experiments have been implemented on a $2.8 \mathrm{GHz}$ Pentium D dual processor with $512 \mathrm{MB}$ of memory using visual $\mathrm{C}++$ (version 6.0 ) software. The experimental results are presented on both real and synthetic datasets. We have constructed artificial database check to verify that the proposed algorithm works correctly. Each item is represented by an integer number to perform experiments more conveniently. Thus, a transaction in check is a collection of integer numbers separated by commas. The dataset retail [14] is obtained from an anonymous Belgian retail supermarket store. Also, we have generated a synthetic dataset SD100000. We present some characteristics of these datasets in Table 3.

The symbols NT, AFI, ALT and NI denote the number of transactions, average frequency of an item, average length of a transaction and number of items in the dataset, respectively. Each of the above datasets has been divided into 10 databases for the purpose of carrying out experiments. These databases are called input databases. The proposed work is based on the frequent itemsets in the input databases. There are many algorithms $[10,17,21]$ for mining frequent itemsets in a database. Thus, there exist many implementations [13] of mining frequent itemsets in a database.

\begin{tabular}{lcccc}
\hline Dataset & NT & ALT & AFI & NI \\
\hline $\operatorname{check}(C)$ & 40 & 3.03 & 3.10 & 39 \\
$\operatorname{retail}(R)$ & 88,162 & 11.31 & 99.71 & 10,000 \\
$\operatorname{SD} 100000(S)$ & $1,00,000$ & 15.11 & 151.10 & 10,000 \\
\hline
\end{tabular}

Table 3. Dataset characteristics. 
The dataset check consists of 40 transactions. The input databases obtained from check are given as follows:

$$
\begin{aligned}
& C_{0}=\{\{1,4,9,31\},\{1,4,7,10,50\},\{1,4,10,20,24\},\{1,4,10,23\}\} ; \\
& C_{1}=\{\{1,4,10,34\},\{1,3,44\},\{1,2,3,10,20,44\},\{2,3,20,39\}\} ; \\
& C_{2}=\{\{2,3,20,44\},\{2,3,45\},\{2,3,44,50\},\{2,3,20,44,50\}\} ; \\
& C_{3}=\{\{3,44\},\{3,19,50\},\{5,7,21\},\{5,8\}\} ; \\
& C_{4}=\{\{5,41,45\},\{5,49\},\{5,7,21\},\{5,11,21\}\} ; \\
& C_{5}=\{\{6,41\},\{6,15,19\},\{11,12,13\},\{11,21,49\}\} ; \\
& C_{6}=\{\{11,19\},\{21\},\{21,24,39\},\{22,26,38\}\} ; \\
& C_{7}=\{\{22,30,31\},\{24,35\},\{25,39,49\},\{26,41,46\}\} ; \\
& C_{8}=\{\{30,32,42\},\{32,49\},\{41,45,59\},\{42,45\}\} ; \\
& C_{9}=\{\{42,47\},\{45,49\},\{47,48,49\},\{49\}\} .
\end{aligned}
$$

The input databases obtained from retail and SD100000 are named as $R_{i}$ and $S_{i}$, $i=0,1, \ldots, 9$. For the purpose of mining input databases, we have implemented an a priori algorithm [10], since it is simple and popular. Some characteristics of these input databases (DB) are presented in Tables 4 and 5.

The global exceptional frequent itemsets corresponding to check, retail and SD100000 are presented in Tables 6, 7 and 8, respectively. The choices of values of different parameters are dependent on data as well as the requirements of decision-making processes. For mining a parse database, we need to lower $\alpha$ (minimum support) significantly to get some output of a data mining algorithm. $\gamma_{1}$ being the threshold of low extraction, is expected to be around 0.4. This implies that if an itemset is extracted from less than four out of ten data sources, one could call it low frequent itemset. But, the value of high support $\left(\mu_{2}\right)$ is very much dependent on the data to be mined as well as the requirement. For example, support value 0.1 of an itemset in a dense database might not be high. But, it could be very high in a parse database.

A global exceptional frequent itemset might not be supported with equal degree from the source databases. For example, the global exceptional frequent itemset $\{5\}$ has been extracted from databases $C_{3}$ and $C_{4}$. But, the database $C_{4}$ reports itemset $\{5\}$ exceptionally high. Each available itemset in a local database is greater than or equal to 0.25 , since there are only four transactions available in each of the input databases $C_{0}, C_{1}, \ldots, C_{9}$.

We observe that some databases do not report any global exceptional frequent itemsets. On the contrary, some other databases are the source of many global 


\begin{tabular}{cccccccccc}
\hline DB & NT & ALT & AFI & NI & DB & NT & ALT & AFI & NI \\
\hline$R_{0}$ & 9,000 & 11.24 & 12.07 & 8,384 & $R_{5}$ & 9,000 & 10.86 & 16.71 & 5,847 \\
$R_{1}$ & 9,000 & 11.21 & 12.26 & 8,225 & $R_{6}$ & 9,000 & 11.20 & 17.42 & 5,788 \\
$R_{2}$ & 9,000 & 11.34 & 14.60 & 6,990 & $R_{7}$ & 9,000 & 11.16 & 17.35 & 5,788 \\
$R_{3}$ & 9,000 & 11.49 & 16.66 & 6,206 & $R_{8}$ & 9,000 & 11.99 & 18.69 & 5,777 \\
$R_{4}$ & 9,000 & 10.96 & 16.04 & 6,148 & $R_{9}$ & 7,162 & 11.69 & 15.35 & 5,456 \\
\hline
\end{tabular}

Table 4. The characteristics of databases obtained from retail.

\begin{tabular}{cccccccccc}
\hline DB & NT & ALT & AFI & NI & DB & NT & ALT & AFI & NI \\
\hline$S_{0}$ & 10,000 & 16.01 & 17.08 & 9,371 & $S_{5}$ & 10,000 & 15.77 & 15.95 & 9,886 \\
$S_{1}$ & 10,000 & 15.23 & 16.16 & 9,426 & $S_{6}$ & 10,000 & 16.69 & 19.58 & 8,524 \\
$S_{2}$ & 10,000 & 13.93 & 17.35 & 8,031 & $S_{7}$ & 10,000 & 14.93 & 15.77 & 9,468 \\
$S_{3}$ & 10,000 & 12.55 & 14.69 & 8,541 & $S_{8}$ & 10,000 & 15.01 & 16.95 & 8,856 \\
$S_{4}$ & 10,000 & 17.56 & 19.05 & 9,003 & $S_{9}$ & 10,000 & 13.97 & 15.31 & 9,119 \\
\hline
\end{tabular}

Table 5. The characteristics of databases obtained from SD100000.

\begin{tabular}{cccc}
\hline itemset & ssupp & sources & exceptional sources \\
\hline$\{1\}$ & 0.175 & $C_{0}, C_{1}$ & $C_{0}$ \\
$\{2\}$ & 0.175 & $C_{1}, C_{2}$ & $C_{2}$ \\
$\{3\}$ & 0.200 & $C_{1}, C_{2}, C_{3}$ & $C_{1}, C_{2}$ \\
$\{5\}$ & 0.150 & $C_{3}, C_{4}$ & $C_{4}$ \\
$\{44\}$ & 0.150 & $C_{1}, C_{2}, C_{3}$ & $C_{1}, C_{2}$ \\
$\{2,3\}$ & 0.150 & $C_{1}, C_{2}$ & $C_{2}$ \\
$\{3,44\}$ & 0.150 & $C_{1}, C_{2}, C_{3}$ & $C_{2}, C_{3}$ \\
$\{4\}$ & 0.125 & $C_{0}, C_{1}$ & $C_{0}$ \\
$\{10\}$ & 0.125 & $C_{0}, C_{1}$ & $C_{0}$ \\
$\{20\}$ & 0.125 & $C_{0}, C_{1}, C_{2}$ & $C_{1}, C_{2}$ \\
\hline
\end{tabular}

Table 6. Global exceptional frequent itemsets in $\left\{C_{0}, C_{1}, \ldots, C_{9}\right\}$ at $\alpha=0.05$, $\gamma_{1}=0.4$ and $\mu_{2}=0.09$ (top 10). 


\begin{tabular}{cccc}
\hline itemset & ssupp & sources & exceptional sources \\
\hline$\{2,6,9\}$ & 0.102 & $R_{0}, R_{1}$ & $R_{0}$ \\
$\{2,9,41\}$ & 0.103 & $R_{0}, R_{1}, R_{7}$ & $R_{0}, R_{7}$ \\
$\{6,9,41\}$ & 0.107 & $R_{0}, R_{1}, R_{3}$ & $R_{0}, R_{1}$ \\
$\{8,9,271\}$ & 0.102 & $R_{0}$ & $R_{0}$ \\
$\{9,41,48\}$ & 0.102 & $R_{1}$ & $R_{1}$ \\
\hline
\end{tabular}

Table 7. Global exceptional frequent itemsets in $\left\{R_{0}, R_{1}, \ldots, R_{9}\right\}$ at $\alpha=0.02$, $\gamma_{1}=0.4$ and $\mu_{2}=0.1$.

\begin{tabular}{cccc}
\hline itemset & ssupp & sources & exceptional sources \\
\hline$\{22\}$ & 0.247 & $S_{0}, S_{5}, S_{6}$ & $S_{0}$ \\
$\{32\}$ & 0.208 & $S_{2}, S_{4}, S_{5}$ & $S_{4}, S_{5}$ \\
$\{45\}$ & 0.185 & $S_{3}, S_{4}, S_{5}$ & $S_{3}$ \\
$\{4\}$ & 0.167 & $S_{2}, S_{5}$ & $S_{5}$ \\
$\{49\}$ & 0.161 & $S_{4}, S_{9}$ & $S_{4}$ \\
$\{22,32\}$ & 0.158 & $S_{0}, S_{2}, S_{5}$ & $S_{0}, S_{2}$ \\
$\{22,45\}$ & 0.152 & $S_{0}, S_{4}, S_{5}$ & $S_{0}, S_{4}$ \\
$\{22,4\}$ & 0.132 & $S_{0}, S_{2}, S_{5}$ & $S_{0}, S_{5}$ \\
$\{22,49\}$ & 0.130 & $S_{0}, S_{4}$ & $S_{4}$ \\
$\{32,45\}$ & 0.121 & $S_{2}, S_{4}$ & $S_{2}$ \\
\hline
\end{tabular}

Table 8. Global exceptional frequent itemsets in $\left\{S_{0}, S_{1}, \ldots, S_{9}\right\}$ at $\alpha=0.02$, $\gamma_{1}=0.4$ and $\mu_{2}=0.05$ (top 10).

exceptional frequent itemsets. In Tables 9,10 and 11, we present the distributions of global exceptional frequent itemsets in $\left\{C_{0}, C_{1}, \ldots, C_{9}\right\},\left\{R_{0}, R_{1}, \ldots, R_{9}\right\}$ and $\left\{S_{0}, S_{1}, \ldots, S_{9}\right\}$, respectively. In Table 9 , we notice that three out of ten databases are not a source of any global exceptional frequent itemset.

The distribution of global exceptional frequent itemsets in $\left\{R_{0}, R_{1}, \ldots, R_{9}\right\}$ is different from that in $\left\{C_{0}, C_{1}, \ldots, C_{9}\right\}$. In Table 10 , we notice that seven out of ten databases are not a source of any exceptional global frequent itemset. In Table 11, we observe that most of the databases are a source of some exceptional global frequent itemsets.

Also, we have studied the number of global exceptional frequent itemsets in multi-databases at different $\gamma_{1}$. As we increase $\gamma_{1}$, we allow more frequent itemsets to be global exceptional. In Figures 1, 2 and 3, we study the relationship 


\begin{tabular}{lcccccccccc}
\hline database & $C_{0}$ & $C_{1}$ & $C_{2}$ & $C_{3}$ & $C_{4}$ & $C_{5}$ & $C_{6}$ & $C_{7}$ & $C_{8}$ & $C_{9}$ \\
\hline $\begin{array}{l}\text { number of sources of global } \\
\text { exceptional frequent item- } \\
\text { sets }\end{array}$ & 7 & 8 & 10 & 4 & 2 & 1 & 1 & 0 & 0 & 0 \\
\hline
\end{tabular}

Table 9. Distribution of exceptional source for global exceptional frequent itemsets in $\left\{C_{0}, C_{1}, \ldots, C_{9}\right\}$ at $\alpha=0.05, \gamma_{1}=0.4$ and $\mu_{2}=0.09$.

\begin{tabular}{lcccccccccc}
\hline database & $R_{0}$ & $R_{1}$ & $R_{2}$ & $R_{3}$ & $R_{4}$ & $R_{5}$ & $R_{6}$ & $R_{7}$ & $R_{8}$ & $R_{9}$ \\
\hline $\begin{array}{l}\text { number of sources of global } \\
\text { exceptional frequent item- } \\
\text { sets }\end{array}$ & 4 & 2 & 0 & 0 & 0 & 0 & 0 & 1 & 0 & 0 \\
\hline
\end{tabular}

Table 10. Distribution of global exceptional frequent itemsets in $\left\{R_{0}, R_{1}, \ldots, R_{9}\right\}$ at $\alpha=0.02, \gamma_{1}=0.4$ and $\mu_{2}=0.1$.

\begin{tabular}{lcccccccccc}
\hline database & $S_{0}$ & $S_{1}$ & $S_{2}$ & $S_{3}$ & $S_{4}$ & $S_{5}$ & $S_{6}$ & $S_{7}$ & $S_{8}$ & $S_{9}$ \\
\hline $\begin{array}{l}\text { number of sources of global } \\
\text { exceptional frequent item- } \\
\text { sets }\end{array}$ & 11 & 0 & 6 & 9 & 1 & 5 & 7 & 3 & 3 & 5 \\
\hline
\end{tabular}

Table 11. Distribution of global exceptional frequent itemsets in $\left\{S_{0}, S_{1}, \ldots, S_{9}\right\}$ at $\alpha=0.02, \gamma_{1}=0.4$ and $\mu_{2}=0.05$.

between $\gamma_{1}$ and the number of global exceptional frequent itemsets in multiple databases.

We have observed that the number of global exceptional frequent itemsets do not vary much at different $\gamma_{1}$. In fact, there is only one change in both the graphs of Figures 1 and 2.

Also, we have studied the number of global exceptional frequent itemsets in multiple databases at different $\alpha$. We present experimental results in Figures 4, 5 and 6 . The number of global exceptional frequents in $\left\{C_{0}, C_{1}, \ldots, C_{9}\right\}$ remains fixed at 21 over different $\alpha$.

But, we find a different trend with respect to the number of global exceptional frequent itemsets in $\left\{R_{0}, R_{1}, \ldots, R_{9}\right\}$. At lower and upper values of $\alpha$, the number of global exceptional frequent itemsets is 0 . Again, we get some global exceptional frequent itemsets for some middle values of $\alpha$. Thus, there is no fixed relationship between the number of global exceptional frequent itemsets and $\alpha$. 


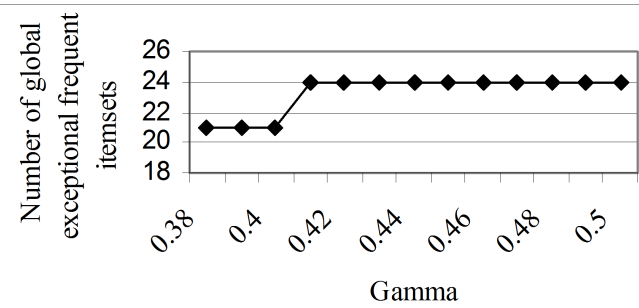

Figure 1. Number of global exceptional frequent itemsets in $\left\{C_{0}, C_{1}, \ldots, C_{9}\right\}$ at $\alpha=0.05$, and $\mu_{2}=0.1$.

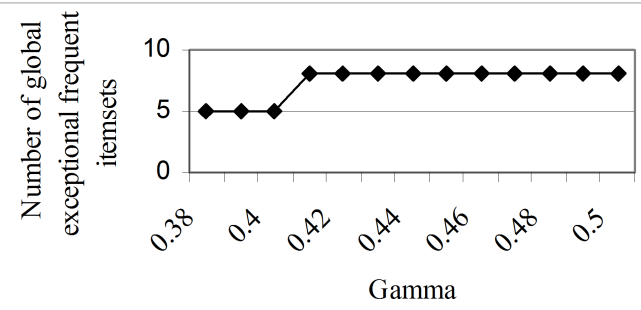

Figure 2. Number of global exceptional frequent itemsets in $\left\{R_{0}, R_{1}, \ldots, R_{9}\right\}$ at $\alpha=0.02$, and $\mu_{2}=0.1$.

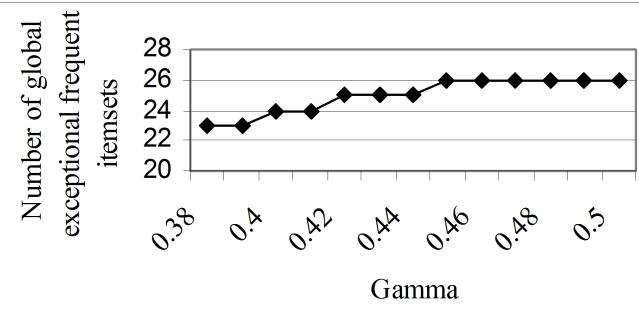

Figure 3. Number of global exceptional frequent itemsets in $\left\{S_{0}, S_{1}, \ldots, S_{9}\right\}$ at $\alpha=0.02$, and $\mu_{2}=0.05$. 


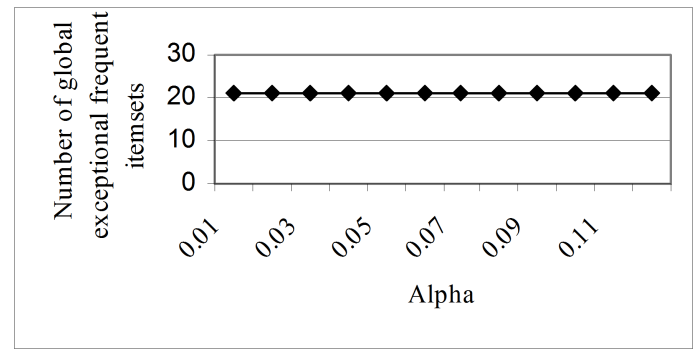

Figure 4. Number of global exceptional frequent itemsets in $\left\{C_{0}, C_{1}, \ldots, C_{9}\right\}$ at $\gamma_{1}=0.4$, and $\mu_{2}=0.1$.

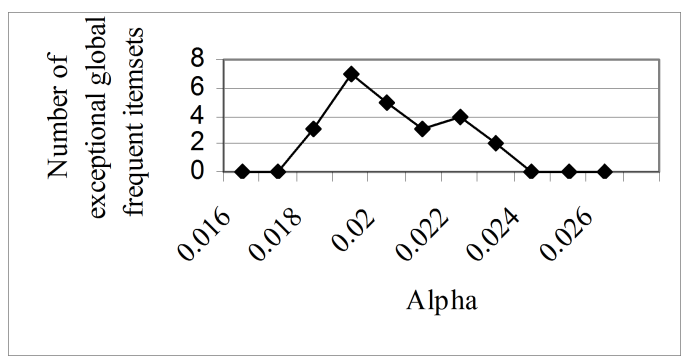

Figure 5. Number of global exceptional frequent itemsets in $\left\{R_{0}, R_{1}, \ldots, R_{9}\right\}$ at $\gamma_{1}=0.4$, and $\mu_{2}=0.1$.

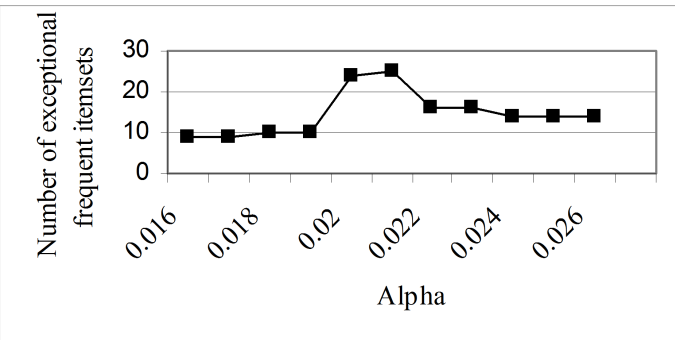

Figure 6. Number of global exceptional frequent itemsets in $\left\{S_{0}, S_{1}, \ldots, S_{9}\right\}$ at $\gamma_{1}=0.4$, and $\mu_{2}=0.05$. 


\begin{tabular}{lcccll}
\hline Databases & $\alpha$ & $\gamma_{1}$ & $\mu_{2}$ & $(\mathrm{AE}, \mathrm{ANT}, \mathrm{ALT}, \mathrm{ANI})$ & $(\mathrm{ME}, \mathrm{ANT}, \mathrm{ALT}, \mathrm{ANI})$ \\
\hline$C_{0}, C_{1}, \ldots, C_{9}$ & 0.05 & 0.4 & 0.1 & $(0,4,3.025,8.4)$ & $(0,4,3.025,8.4)$ \\
$R_{0}, R_{1}, \ldots, R_{9}$ & 0.02 & 0.4 & 0.1 & $(0.064,8816.2$, & $(0.085,8816.2$, \\
& & & & $11.306,5882.1)$ & $11.306,5882.1)$ \\
$S_{0}, S_{1}, \ldots, S_{9}$ & 0.02 & 0.4 & 0.05 & $(0.052,10000.0$, & $(0.075,10000.0$, \\
& & & & $15.11,9022.5)$ & $15.11,9022.5)$ \\
\hline
\end{tabular}

Table 12. Errors of the experiments at a given value of tuple $(\alpha, \gamma, \mu)$.

Also, we have calculated the error of the experiments. In Table 12, we present the error of the experiments at a given value of tuple $(\alpha, \gamma, \mu)$.

\subsection{Comparison with the Existing Algorithm}

In this section, we compare IdentifyExPattern [28] and Type-II-ExceptionalFrequent-Itemset-Synthesis. We analyze and compare these two algorithms on the basis of experiments conducted on the following two aspects: (i) average error versus $\alpha$, and (ii) synthesizing time versus number of databases.

\section{Average Error}

We have calculated the AEs at different $\alpha$ to study the relationship between them. Experimental results are presented in Figures 7, 8 and 9. We observe that there is no fixed relationship between $\mathrm{AE}$ and $\alpha$.

In both cases, algorithm Type-II-Exceptional-Frequent-Itemset-Synthesis performs better than algorithm IdentifyExPattern. In dataset check, the global exceptional frequent itemsets are not uniformly distributed. The global exceptional frequent itemsets appear only in few databases, while they remain absent in the remaining databases. Each frequent itemset is reported from all the data sources. So, the error of synthesizing a frequent itemset in $\left\{C_{0}, C_{1}, \ldots, C_{9}\right\}$ is zero. At support level 0.1, all itemsets become frequent. Thus, the AE of an experiment becomes 0 .

Figures 8 and 9 show small differences between the AEs. When a global decision is based on the approximate patterns in multiple large databases, such differences between the AEs using different algorithms are not significant. But, it does matter when the decisions are based on high quality of patterns from multiple large databases. 


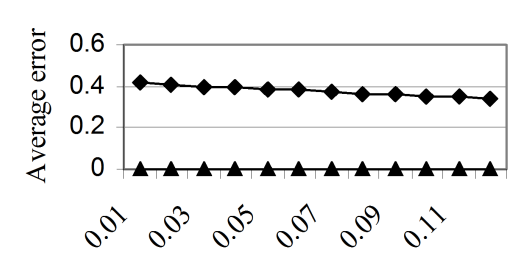

$\neg$ AE using algm ExceptionalFrequentItemset-

$\multimap$ AE using algm Identify ExPattern

Alpha

Figure 7. Average error versus $\alpha$ for check at $\gamma_{1}=0.4$, and $\mu_{2}=0.1$.

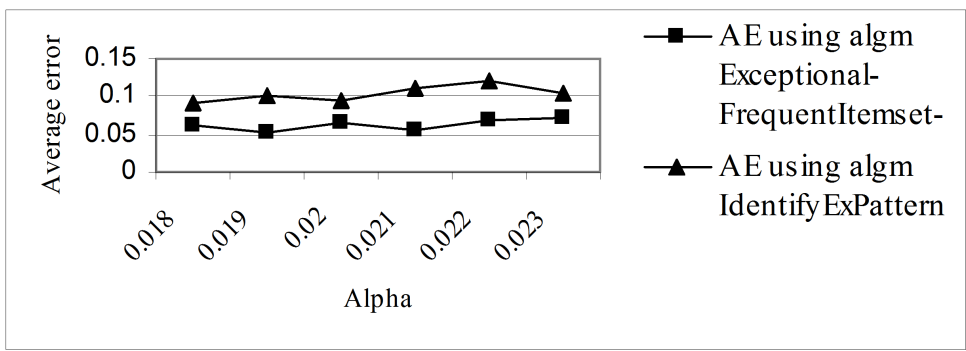

Figure 8. Average error versus $\alpha$ for retail at $\gamma_{1}=0.4$, and $\mu_{2}=0.1$.

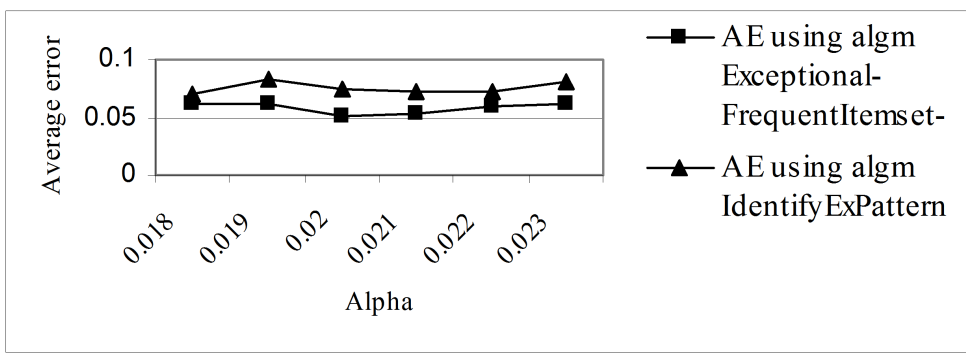

Figure 9. Average error versus $\alpha$ for SD100001 at $\gamma_{1}=0.4$, and $\mu_{2}=0.05$. 


\section{Synthesizing Time}

Also, we have calculated the time for synthesizing global exceptional frequent itemsets by varying the number of databases. In Figures 10, 11 and 12, we show time (in ms) required for synthesizing global exceptional frequent itemsets in multiple databases. In case of the experiment conducted on $\left\{C_{0}, C_{1}, \ldots, C_{9}\right\}$, we observe that the synthesizing time does not increase as the number of databases increases. This is due to the fact that the size of each of the databases is very small. In fact, the time required for synthesizing global exceptional frequent itemsets in $\left\{C_{0}, C_{1}, \ldots, C_{9}\right\}$ is $0 \mathrm{~ms}$, for both the algorithms.

From the results presented in Figures 10, 11 and 12, we could conclude that algorithm Type-II-Exceptional-Frequent-Itemset-Synthesis executes faster than algorithm IdentifyExPattern. Also, it validates the theoretical result (see Theorems 1 and 2). In general, the time for synthesizing global exceptional frequent itemsets either remains the same or increases as the number of databases increases.

\section{Conclusion}

The synthesis of global exceptional patterns is an important component of a multidatabase mining system. Many corporate decisions of a multi-branch company would depend on global exceptional patterns in branch databases. We have identified the short-comings of the existing concepts and the algorithm to identify global exceptional patterns. We have proposed notions of type I and type II global exceptional frequent itemsets. Also, we have proposed notions of type I and type II global exceptional association rules. We have introduced the notion of exceptional sources for a global exceptional frequent itemset. The proposed algorithm identifies type II global exceptional frequent itemsets and their exceptional sources in multiple databases. We have also compared the proposed algorithm with the existing algorithm. The proposed algorithm performs better than the existing algorithm on the following aspects: (i) error of the experiment and (ii) execution time. We have observed that the proposed algorithm executes faster when the number of databases increases. Also, we have shown theoretically that the proposed algorithm executes faster than the existing algorithm. The proposed solution is simple and effective in synthesizing type II global exceptional frequent itemsets in multiple databases. 


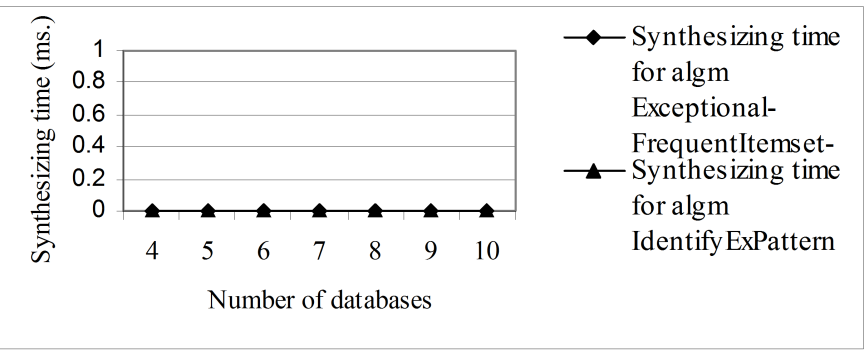

Figure 10. Synthesizing time versus number of databases obtained from check at $\gamma_{1}=0.4$, and $\mu_{2}=0.1$.

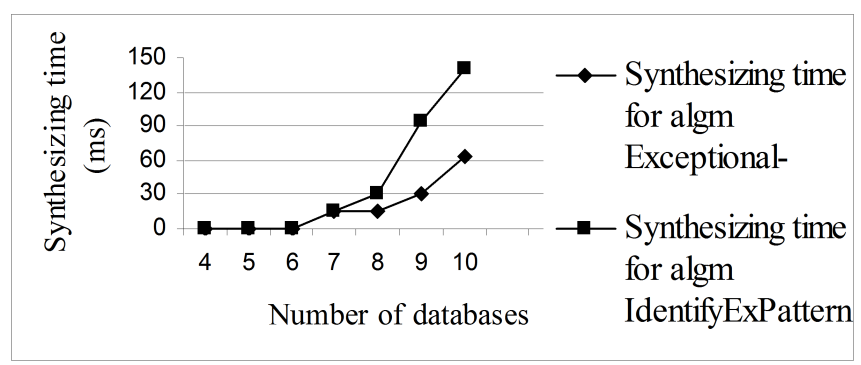

Figure 11. Synthesizing time versus number of databases obtained from retail at $\alpha=0.02, \gamma_{1}=0.4$, and $\mu_{2}=0.1$.

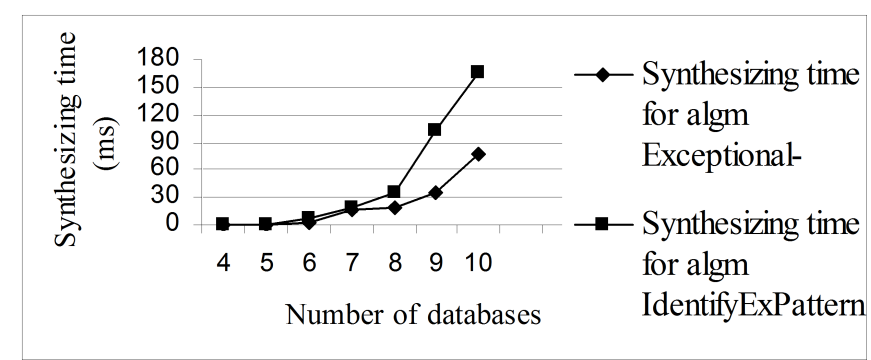

Figure 12. Synthesizing time versus number of databases obtained from SD100001 at $\alpha=0.02, \gamma_{1}=0.4$, and $\mu_{2}=0.05$. 


\section{Bibliography}

[1] A. Adhikari, P. Ramachandrarao and W. Pedrycz, Developing Multi-Database Mining Applications, Springer, 2010.

[2] A. Adhikari and P. R. Rao, Efficient clustering of databases induced by local patterns, Decis. Support Syst. 44 (2008), 925-943.

[3] A. Adhikari and P. R. Rao, Mining conditional patterns in a database, Pattern Recognit. Lett. 29 (2008), 1515-1523.

[4] A. Adhikari and P. R. Rao, Synthesizing heavy association rules from different real data sources, Pattern Recognit. Lett. 29 (2008), 59-71.

[5] J. Adhikari, P. R. Rao and W. Pedrycz, Mining icebergs in time-stamped databases, in: Indian International Conferences on Artificial Intelligence (2011), 639-658.

[6] A. Adhikari, P. R. Rao, B. Prasad and J. Adhikari, Mining multiple large data sources, Int. Arab J. Inf. Tech. 7 (2010), 241-249.

[7] J.-M. Adamo, Data Mining for Association Rules and Sequential Patterns: Sequential and Parallel Algorithms, Springer, 2001.

[8] R. Agrawal, T. Imielinski and A. Swami, Mining association rules between sets of items in large databases, in: Proceedings of ACM SIGMOD Conf. Management of Data (1993), 207-216.

[9] R. Agrawal and J. Shafer, Parallel mining of association rules, IEEE Trans. Knowl. Data Eng. 8 (1999), 962-969.

[10] R. Agrawal and R. Srikant, Fast algorithms for mining association rules, in: Proceedings of the International Conference on Very Large Data Bases (1994), 487-499.

[11] J. Chattratichat, J. Darlington,, M. Ghanem, Y. Guo, H. Hüning, M. Köhler, J. Sutiwaraphun, H. W. To and D. Yang, Large scale data mining: Challenges, and responses, in: Proceedings of the Third International Conference on Knowledge Discovery and Data Mining (1997), 143-146.

[12] D. Cheung, V. Ng, A. Fu and Y. Fu, Efficient mining of association rules in distributed databases, IEEE Trans. Knowl. Data Eng. 8 (1996), 911-922.

[13] FIMI 2004, http://fimi.cs.helsinki.fi/src/.

[14] Frequent Itemset Mining Dataset Repository, http://fimi.cs.helsinki.fi/ data/.

[15] J. Galambos and I. Simonelli, Bonferroni-Type Inequalities with Applications, Springer, 1996.

[16] A. Greenfield, Everyware: The Dawning Age of Ubiquitous Computing, New Riders Publishing, 2006.

[17] J. Han, J. Pei and Y. Yiwen, Mining frequent patterns without candidate generation, in: Proceedings ACM SIGMOD Conf. Management of Data (2000), 1-12. 
[18] S. Jaroszewicz and D. A. Simovici, Support approximations using Bonferroni-type inequalities, in: Proceedings of sixth European Conference on Principles of Data Mining and Knowledge Discovery, (2002), 212-223.

[19] H. Kargupta, J. Han, P. S. Yu, R. Motwani and V. Kumar, Next Generation of Data Mining, Springer, 2008.

[20] D. Pavlov, H. Mannila and P. Smyth, Probabilistic models for query approximation with large sparse binary data sets, in: Proceedings of Sixteenth Conference on Uncertainty in Artificial Intelligence (2000), 465-472.

[21] A. Savasere, E. Omiecinski and S. Navathe, An efficient algorithm for mining association rules in large databases, in: Proceedings of the 21st International Conference on Very Large Data Bases (1995), 432-443.

[22] K. Wang, S. Zhou and Y. He, Hierarchical classification of real life documents, in: Proceedings of the 1st (SIAM) International Conference on Data Mining (2001), 1-16.

[23] B. Wilkinson, Grid Computing: Techniques and Applications, CRC Press, 2009.

[24] X. Wu, Y. Wu, Y. Wang and Y. Li, Privacy-aware market basket data set generation: A feasible approach for inverse frequent set mining, in: Proceedings of SIAM International Conference on Data Mining (2005), 103-114.

[25] X. Wu, C. Zhang and S. Zhang, Efficient mining of both positive and negative association rules, ACM Trans. Inform. Syst. 22 (2004), 381-405.

[26] X. Wu, C. Zhang and S. Zhang, Database classification for multi-database mining, Inform. Syst. 30 (2005), 71-88.

[27] X. Wu and S. Zhang, Synthesizing high-frequency rules from different data sources, IEEE Trans. Knowl. Data Eng. 14 (2003), 353-367.

[28] C. Zhang, M. Liu, W. Nie and S. Zhang, Identifying global exceptional patterns in multi-database mining, IEEE Computat. Intell. Bull. 3 (2004), 19-24.

[29] C. Zhang and S. Zhang, Association Rule Mining: Models and Algorithms, Springer, 2002.

[30] S. Zhang and X. Wu, Fundamentals of association rules in data mining and knowledge discovery, Data Min. Knowl. Disc. 1 (2011), 97-116.

[31] S. Zhang, X. Wu and C. Zhang, Multi-database Mining, IEEE Computat. Intell. Bull. 2 (2003), 5-13.

[32] S. Zhang, C. Zhang and X. Wu, Knowledge Discovery in Multiple Databases, Springer, 2004.

[33] S. Zhang, C. Zhang and J. X. Yu, An efficient strategy for mining exceptions in multi-databases, Inform. Sciences 165 (2004), 1-20.

[34] F. Zhao and L. Guibas, Wireless Sensor Networks: An Information Processing Approach, Morgan Kaufmann, 2004. 
Received April 25, 2012.

\section{Author information}

Animesh Adhikari, Department of Computer Science, S. P. Chowgule College, Margao, Goa 403602, India.

E-mail: animeshadhikari@yahoo.com 